\title{
Dendrimer-Stabilized Titanate Nanowire Dispersions as Potential Nanocarriers
}

\author{
Marko Pavlovic, ${ }^{\dagger}$ Monika Adok-Sipiczki, ${ }^{\dagger}$ Endre Horváth, ${ }^{\ddagger}$ Tamás Szabó, ${ }^{\S}$ László Forró, ${ }^{\ddagger}$ \\ and Istvan Szilagyi* ${ }^{\dagger}$ \\ ${ }^{\dagger}$ Department of Inorganic and Analytical Chemistry, University of Geneva, 30 Quai Ernest-Ansermet, CH-1205 Geneva, Switzerland \\ ${ }^{\ddagger}$ Laboratory of Physics of Complex Matter, École Polytechnique Fédérale de Lausanne, CH-1015 Lausanne, Switzerland \\ ${ }^{\S}$ Department of Physical Chemistry and Materials Science, University of Szeged, H-6720 Szeged, Hungary
}

ABSTRACT: The effect of poly(amido amine) (PAMAM) dendrimers of generations G2, G6, and G10 on the dispersion stability of titanate nanowires (TiONWs) as potential nanocarriers was clarified in order to develop biocompatible delivery systems. The PAMAMs adsorbed strongly on the oppositely charged TiONWs even under slightly alkaline conditions where the macromolecules possess low amounts of charged groups and they were able to reverse the charge of the particles. This process resulted in unstable dispersions near the dose where the charge reversal occurred, while stable samples were observed away from it. No generation dependence was found on the aggregation and charging behavior at low ionic strengths. Although heterogeneous adsorption of the dendrimers led to attractive patch-charge interactions at intermediate doses,

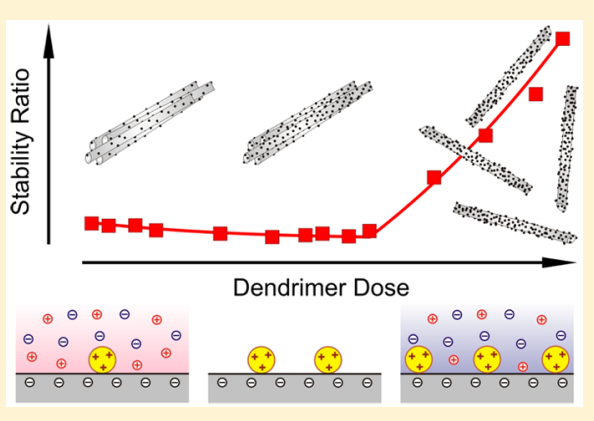
interparticle forces of electrostatic origin described by Derjaguin and Landau, Verwey, and Overbeek (DLVO) played the major role in colloidal stability. The nanowires were found to adopt a face-to-face orientation within the aggregates, giving rise to the formation of well-defined bundles. Highly stable dispersions were observed once the TiONWs were coated with PAMAM dendrimers of higher generations even at elevated ionic strengths.

\section{INTRODUCTION}

Layered materials have anisotropic structure, and they have recently attracted widespread interest due to their advantageous structural and mechanical properties as well as their potential application as a source of unilamellar nanosheets. ${ }^{1,2}$ Among such lamellar compounds, elongated titanate derivatives represent a new class of materials which are widely used as building blocks in sensors, nanocarriers, and potential catalysts. $^{3-9}$ Accordingly, nanocomposites consisting of layered titanates and hemoglobin showed high biocatalytic activity even in nonaqueous media ${ }^{10}$ and reduced graphite oxide supported by layered titania was developed and used as a mediator-free biosensor. ${ }^{11}$ In addition, immobilization of horseradish peroxidase enzyme on layered titanate afforded a catalyst of good recyclability. ${ }^{12}$ More recently, other titanate derivatives such as nanowires, ${ }^{13}$ nanotubes, ${ }^{14}$ and nanoflowers ${ }^{15}$ were applied in drug delivery systems. The titanate nanowires (TiONWs) decorated with anatase crystals showed high loading ability for protein-based drugs, while nanotubes entered into cells successfully by endocytosis.

Most of the biomedical applications require functionalization of the titanate surfaces in order to prevent the aggregation of the particles in the living systems and to achieve well-controlled drug release. Moreover, titanate-based nanofilaments were found to be cytotoxic and thus their surface must be modified with biocompatible compounds. ${ }^{16}$ To deal with these challenges, titanate nanotubes were coated with polyelectrolytes, ${ }^{17}$ biodegradable polymers, ${ }^{18}$ or inorganic nanoparticles. ${ }^{19}$
For instance, poly(ethylene imine)-coated particles showed better dispersion properties as the surface charge of the nanocarrier could be tuned easily by the $\mathrm{pH}^{17}$ The dispersion stability of TiONWs was also investigated by aggregation kinetic measurements in the presence of polyelectrolytes below and above the point of zero charge. ${ }^{20-22}$ They revealed that the colloidal stability of the samples can be appropriately tuned by varying the concentration of the polyelectrolyte, $\mathrm{pH}$, or ionic strength and that full polyelectrolyte coating resulted in highly stable aqueous dispersions.

Although these studies report polyelectrolytes as appropriate stabilizing agents, further research has to be performed in order to clarify their toxicity and to find specific ones for targeted drug delivery processes. Promising candidates to meet such requirements are the dendrimers. For instance, poly(amido amine) (PAMAM) dendrimers are widely used in biomedical applications. $^{23-26}$ Enzyme mimicking catalytic systems were also developed using PAMAMs, ${ }^{27}$ and their composite materials were successfully applied as gene delivery vectors. ${ }^{25}$

PAMAM dendrimers are monodisperse, symmetrically branched macromolecules containing an ethylenediamine core and primary amines on the outer spheres. They are connected with oligoamide chains, and they double their molecular weight approximately by increasing the number of generations. ${ }^{26}$ The

Received: September 8, 2015

Revised: October 6, 2015

Published: October 16, 2015 
primary and tertiary amines can be protonated at appropriately low $\mathrm{pH}$ where the dendrimers become positively charged. Their protonation under different experimental conditions, ${ }^{28}$ along with other fundamental properties such as self-diffusion ${ }^{29}$ and their reversible swelling process, ${ }^{30}$ was also investigated in detail. Toxicity of PAMAMs depends on the generation number and their concentration in the living systems; ${ }^{23,26}$ however, they were found to be biocompatible both in the bare stage $^{31}$ and in composites. ${ }^{25,32}$

PAMAM dendrimers are ideal building blocks for a wide range of composite materials; therefore, their interaction with nanomaterials was extensively studied. ${ }^{33}$ Accordingly, intercalation in layered clays, ${ }^{34}$ their association with other macromolecules to form interpolyelectrolyte complexes, ${ }^{35}$ and their ability to stabilize transition metal nanoclusters ${ }^{36}$ have been reported. Adsorption experiments were also carried out on silica, ${ }^{37,38}$ graphite, $^{39}$ and gold ${ }^{40}$ surfaces using reflectometry, atomic force microscopy (AFM), and quartz crystal microbalance, respectively. For silica, it was found that low molecular weight PAMAMs at low $\mathrm{pH}$ and high ionic strength adsorb reversibly on the surface, while the adsorption of high generation ones at high $\mathrm{pH}$ and low ionic strength is completely irreversible.

Interaction of PAMAM dendrimers with colloidal particles was also widely studied to tune interparticle forces and related dispersion stability of the samples. For instance, silver nanoparticles were synthesized and protected against aggregation by adsorbed PAMAM dendrimers. ${ }^{41}$ Detailed investigations were performed on systems containing sulfate modified polystyrene latex particles and PAMAM dendrimers in aqueous dispersions. Electrophoretic and time-resolved dynamic light scattering (DLS) experiments revealed that the molecules strongly adsorb on oppositely charged latexes leading to charge neutralization and subsequent charge reversal at appropriate doses. $^{42}$ The latter process was found to be superstoichiometric since it involves coadsorption of the dendrimer counterions. Unstable dispersions were reported near the charge neutralization point, while the samples were stable far from that dendrimer dose. Two types of interparticle forces were discovered, namely, classical forces described by Derjaguin and Landau, Verwey, and Overbeek (DLVO) and attractive patch-charge interactions due to the heterogeneous surfaces created by the incomplete coverage by the adsorbed PAMAM molecules. Such forces were identified by both the AFM colloidal probe technique and computer simulations using similar latex particles of higher size. ${ }^{43,44}$ More recently, aggregation rate coefficients of these polystyrene particles were determined in the presence of PAMAM dendrimers by AFM and DLS and both experiments yielded very similar values especially for PAMAM of lower generation. ${ }^{45}$

Apart from these results concerning monodisperse spheres as model colloidal particles, no systematic aggregation studies have been published with systems containing titanate nanoparticles of anisotropic structure and PAMAM dendrimers. Given the facts that titanate derivatives are becoming popular nanocarriers in biomedical processes and controlling their aggregation with macromolecules has not been investigated yet in detail, we aimed to study the charging and aggregation of TiONWs in the presence of PAMAM dendrimers to characterize the colloidal stability of this potentially biocompatible delivery system. We were particularly interested in clarifying the effect of molecular weight and ionic strength on the colloidal stability and structure of aggregates using electrophoresis, DLS, and transmission electron microscopy (TEM).

\section{EXPERIMENTAL SECTION}

Materials. The TiONWs were synthesized by a two-step hydrothermal method from anatase (Sigma-Aldrich) which was reacted with concentrated $\mathrm{NaOH}$ (Sigma-Aldrich) solution at $130{ }^{\circ} \mathrm{C}$. In the next step, the intermediate product was treated with concentrated $\mathrm{KOH}$ (Sigma-Aldrich) solution followed by heating at $200{ }^{\circ} \mathrm{C}$ for 1 day. The latter post-treatment process should enhance the stability in morphology and result in a better crystallinity which leads to an excellent long-term reproducibility in the scattering parameters of the nanowires. The white product was then filtered off and washed extensively with ultrapure water produced by a Milli-Q device (Millipore). The TiONWs were dried and suspended in water. The TiONWs have a specific surface area of $186 \mathrm{~m}^{2} / \mathrm{g}$ as determined by nitrogen adsorption experiments using the BET method. For more details, see our previous publications. $^{20,22}$ Similar to other titanium(IV) oxides, ${ }^{46,47}$ the charge of TiONWs strongly depends on the $\mathrm{pH}$. They are negatively charged under alkaline conditions with a surface charge density of $-8.2 \mathrm{mC} / \mathrm{m}^{2}$ at $\mathrm{pH} 9$ as reported earlier. ${ }^{22}$

PAMAM dendrimers (Dendritech) were purchased in aqueous solutions and used without further purification. The characteristic size values for all generations used are shown in Table 1 . The degree of protonation varies only slightly with the generation at $\mathrm{pH} 9$ which was used throughout the experiments. $^{28}$

\section{Table 1. Characteristic Structural Data of the PAMAM} Dendrimers Used in the Present Work ${ }^{a}$

$\begin{array}{cccc}\text { generation } & \text { mol wt }(\mathrm{kg} / \mathrm{mol}) & \operatorname{diam}^{b}(\mathrm{~nm}) & \text { no. primary amine groups } \\ \text { G2 } & 3.3 & 2.9 & 16 \\ \text { G6 } & 58.0 & 6.7 & 256 \\ \text { G10 } & 934.7 & 13.5 & 4096\end{array}$

${ }^{a}$ The values were reported by Dendritech. ${ }^{b}$ Determined by DLS.

The temperature was kept at $25{ }^{\circ} \mathrm{C}$ in the measurements. Glassware, plastic vials, and cuvettes used in the experiments were cleaned with Hellmanex (Hellma) solution and washed thoroughly with ultrapure water.

Methods. The electrophoretic mobilities were measured with a ZetaNano ZS (Malvern) device using plastic capillary cells (Malvern). For sample preparation, PAMAM and $\mathrm{KCl}$ (Sigma-Aldrich) stock solutions were mixed with water to obtain the desired concentration and ionic strength followed by the addition of the TiONW stock to the samples. The procedure resulted in a final sample volume of $5.0 \mathrm{~mL}$ and particle concentration of about $7 \mathrm{mg} / \mathrm{L}$. The electrophoretic mobilities were measured after overnight equilibration of the samples at room temperature. The mobility of each sample was measured five times and averaged.

For determination of the apparent size in time-resolved DLS measurements, the ZetaNano ZS (Malvern) instrument equipped with a $\mathrm{He} / \mathrm{Ne}$ laser and an avalanche photodiode as a detector was used at $173^{\circ}$ scattering angle. The hydrodynamic radii were determined using the cumulant method to fit the correlation function which was accumulated for $30 \mathrm{s.}^{48}$ Square plastic cuvettes (Malvern) of $1 \mathrm{~cm}$ path length were used for the measurements. Regarding sample preparation, dendrimer, $\mathrm{KCl}$ solutions, and ultrapure water 
were mixed to adjust the desired polyelectrolyte dose and ionic strength. The aggregation experiments were initiated by injecting the TiONW stock dispersion which was previously subjected to ultrasonic treatment to mechanically disaggregate the small fraction of agglomerates that may have formed upon aging of the sample. Similar to the electrophoretic measurements, the final particle concentration was about $7 \mathrm{mg} / \mathrm{L}$. This experimental setup allowed us to avoid multiple scattering events and to measure only the translational diffusion coefficient as detailed elsewhere. ${ }^{22}$ In both electrophoretic and aggregation studies, the $\mathrm{pH}$ of stock solutions of dendrimers, $\mathrm{KCl}$, and $\mathrm{TiONW}$ as well as water was adjusted to 9 by $\mathrm{KOH}$ and $\mathrm{HCl}$ (Sigma-Aldrich) prior to mixing them together.

The samples were imaged by TEM using a Tecnai G2 electron microscope (FEI) operating at $120 \mathrm{kV}$. In a typical experiment, a $3 \mu \mathrm{L}$ sample containing approximately $7 \mathrm{mg} / \mathrm{L}$ TiONW, calculated amount of the dendrimers, and $\mathrm{KCl}$ was deposited on a carbon coated 400-mesh copper grid. After $30 \mathrm{~s}$, which was needed for the adsorption of the particles, the liquid phase was blotted off with a filter paper by holding it vertically. The images were then immediately taken from the sample without staining.

\section{RESULTS}

Effects of Dendrimer Generation. Electrophoretic mobilities of TiONWs were measured in the presence of PAMAM dendrimers of generations G2, G6, and G10 (Figure 1a). These macromolecules can be considered as weak polyelectrolytes and thus bear a positive charge at $\mathrm{pH}$ 9, since only about $30 \%$ of the amino groups are protonated under this experimental condition. ${ }^{28}$

In general, the positively charged PAMAMs adsorb on the oppositely charged TiONWs and this is reflected in the increasing mobility values with the dendrimer dose. The adsorption led to charge neutralization at the isoelectric point (IEP) where the overall charge of the TiONW-PAMAM particles is zero. To quantify the contribution of the protonated groups of the dendrimers to the charge balance, one should note that these macromolecules tend to adsorb together with condensed counterions within the structure and, hence, a significant part of the positive PAMAM charge is neutralized by the chloride ions. ${ }^{42}$ Therefore, the effective charge of the macromolecules is lower than the one that can be calculated on the basis of the chemical structure and the protonation equilibria. No trend can be observed with the generation; the IEP values fall within the experimental error. Although a clear dependence was reported on the IEPs earlier in other PAMAM systems, those investigations were carried out with latex particles and under acidic conditions where the PAMAMs are highly charged and significant counterion condensation takes place. $^{42}$

Adding further dendrimers into the samples led to charge reversal which was previously discovered in several particlepolyelectrolyte systems. ${ }^{20-22,42,44,45,49-51}$ The adsorption continues up to the dose at the onset of the adsorption saturation plateau (ASP) which correlates with the establishment of the maximum amount of dendrimers that can adsorb on the particle surface under these experimental conditions. The ASP values fell around $500 \mathrm{mg} / \mathrm{g}$ for all generations within the experimental error. This is much higher than the ASPs determined for other polyelectrolytes adsorbed on TiONWs $(50-100 \mathrm{mg} / \mathrm{g}) .^{20,22}$ Those macromolecules were highly
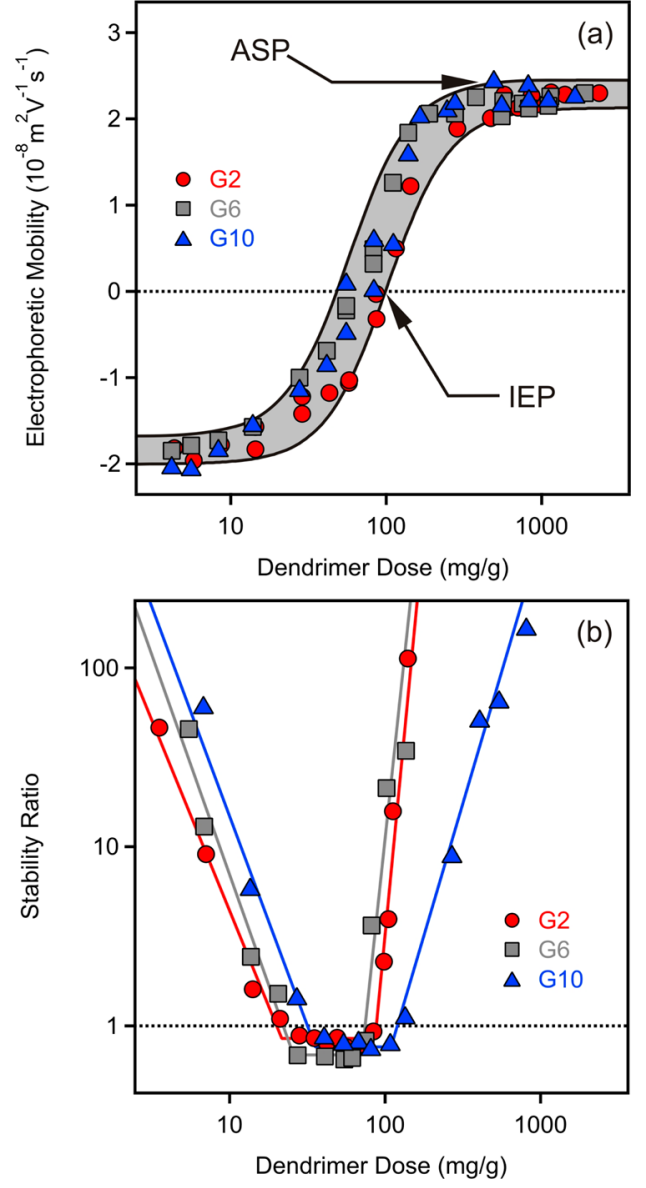

Figure 1. Electrophoretic mobility (a) and stability ratio (b) values as a function of the dendrimer dose measured in dispersions of $7 \mathrm{mg} / \mathrm{L}$ TiONW at $1.0 \mathrm{mM}$ ionic strength for three generations (G2, G6, and G10) of PAMAM. The dose corresponds to milligrams of dendrimer per gram of nanowires. The lines are just to guide the eye.

charged, while the PAMAMs in our systems are only partially protonated and, therefore, the electrostatic repulsion between the adsorbed dendrimers is notably less and a higher amount can be adsorbed on the surface. Another possible explanation for the higher ASPs could be the formation of PAMAM multilayers on the surface, but we do not have unambiguous evidence for such a process. These phenomena are considered generic for all generations of the PAMAMs investigated, because no molecular mass dependence on the charging behavior was observed.

Light scattering has been proved as a suitable tool to follow particle aggregation in dispersions by measuring the apparent hydrodynamic radius under different experimental conditions in a time-resolved experiment. ${ }^{52-54}$ Therefore, time-resolved DLS measurements were carried out to describe the colloidal stability of the systems under the same experimental conditions as in the mobility studies (Figure 1b). Constant values of hydrodynamic radii indicated stable dispersions, while their increase with time was an unambiguous signal for particle aggregation (Figure 2). A linear fit can be performed on the size versus time data, and the slope is proportional to the aggregation rate. Finally, the dispersion stability was expressed in terms of the stability ratio, which is the slope in the case of diffusion controlled aggregation divided by the slope yielded from the actual experiment at the same particle concentration. ${ }^{55}$ 


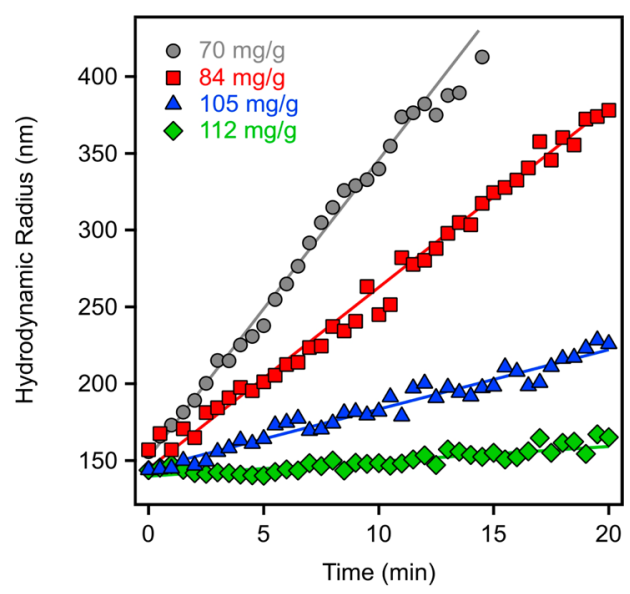

Figure 2. Change in hydrodynamic radius with time in some typical time-resolved DLS experiments with samples containing $7 \mathrm{mg} / \mathrm{L}$ TiONW and different doses of G2 PAMAM dendrimer at $1.0 \mathrm{mM}$ ionic strength. The indicated doses refer to milligrams of dendrimer per gram of nanowires.

The diffusion controlled condition was achieved at $1.0 \mathrm{M} \mathrm{KCl}$ concentration where the surface charges are screened and the particles aggregate rapidly due to attractive van der Waals forces. ${ }^{56}$ Accordingly, stability ratio values close to unity indicate unstable, rapidly aggregating samples, while higher values refer to more stable dispersions.

Figure $1 \mathrm{~b}$ shows the stability ratios for different generations of PAMAMs at low ionic strength. The stability plots for G2 and G6 are almost identical, while it is somewhat different for G10. In all cases, stable dispersions were observed at low dendrimer doses and the stability ratios decreased with increasing concentration. Fast aggregation and unstable systems were discovered near the dose corresponding to the IEP, while the aggregation slowed down at higher PAMAM concentrations. Considering the results of the electrophoretic measurements, such behavior can be explained by the classical DLVO theory developed for charged particles in aqueous dispersions. ${ }^{56}$ Accordingly, the particles are negatively and positively charged at low and high PAMAM doses, respectively (Figure 1a). The surface charge is accompanied by the formation of electric double layers around the particles, and their overlap upon approach gives rise to repulsive interaction forces which can stabilize the sample. However, the TiONWs have an overall charge close to zero around the IEP and the electric double layers vanish, and hence the particles will rapidly aggregate due to the predominating attractive van der Waals forces. A similar origin of interparticle forces was discovered in other particle-polyelectrolyte systems confirmed by direct force measurements using the AFM-based colloidal probe technique. $^{43-45,57}$

However, two phenomena require further clarification. First, the stability ratios in the fast aggregation regimes are systematically lower than 1 . This fact indicates the presence of an additional attractive force which is absent in the reference system ( $\mathrm{TiONW}$ in $1.0 \mathrm{M} \mathrm{KCl}$ ). This force must be induced by the adsorbed PAMAM molecules, and it is of non-DLVO origin. This additional attraction can be explained by patchcharge interactions as follows. The dendrimers adsorb in a heterogeneous fashion on the surface and form positively charged patches. These patches can interact with a negatively charged part of another particle leading to Coulombic attraction and increased aggregation rate. Such patch-charge forces have already been discovered for dendrimers and also for
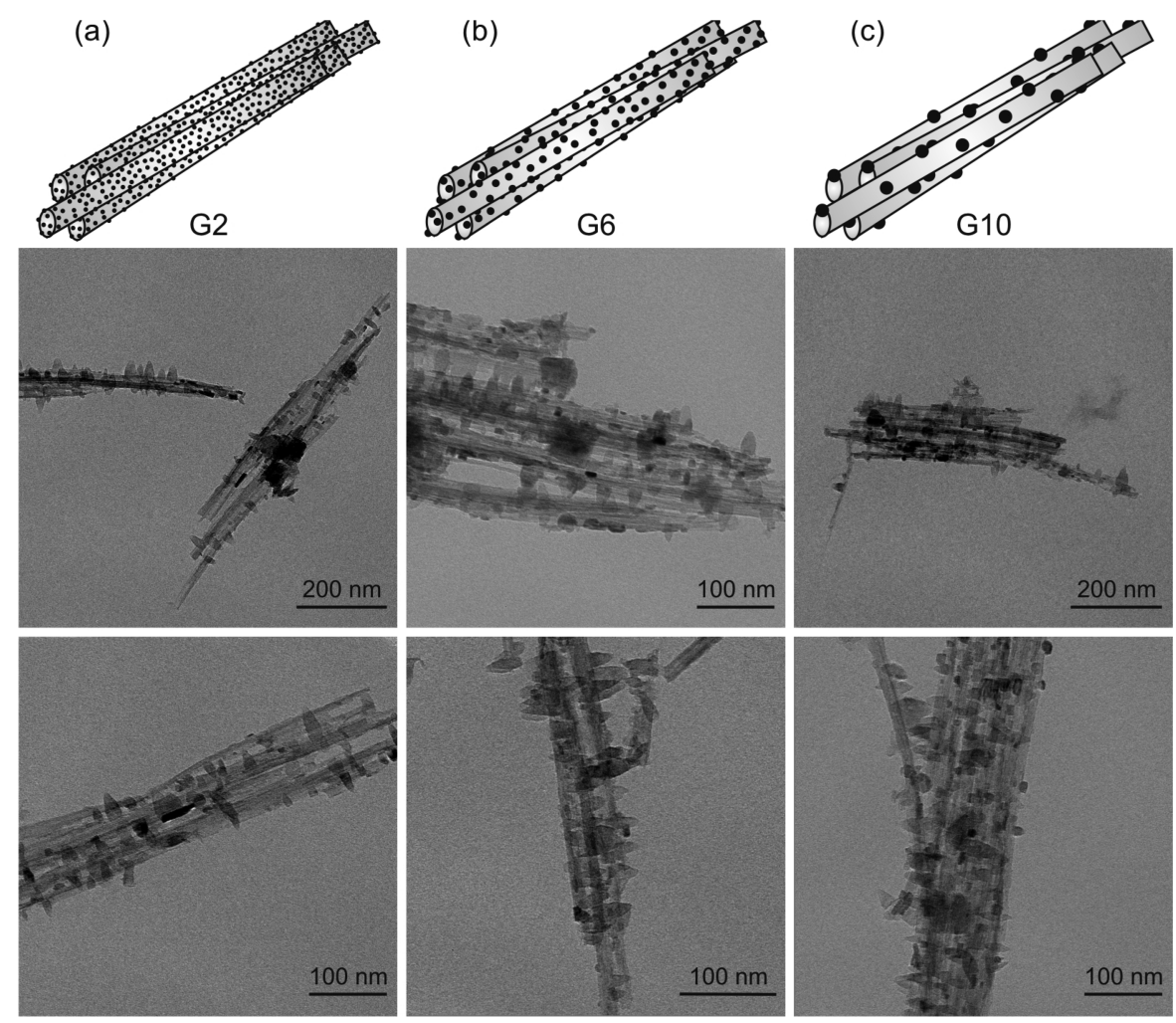

Figure 3. TEM images recorded for systems containing TiONWs and different generations of PAMAMs around the IEP. Dendrimer doses of 57, 55, and $55 \mathrm{mg} / \mathrm{g}$ were applied for G2 (a), G6 (b), and G10 (c), respectively. 
other polyelectrolytes adsorbed on oppositely charged surfaces. $^{20,42,44,57,58}$ Second, the fast aggregation regime is slightly shifted to higher PAMAM doses in the case of G10 dendrimers. A similar finding was reported with latex particles with an even more significant shift. ${ }^{42}$ This effect may originate from the counterion condensation, since higher generation dendrimers bring a considerable amount of negatively charged counterions to the surface and, hence, a part of the PAMAM's charge is already neutralized and a higher amount of dendrimer is required for reaching the IEP. Interestingly, this effect could not be detected in the electrophoretic mobility measurements (Figure 1a), most probably due to the lower sensitivity of that method. Obviously the shift is small and it would be more pronounced if the PAMAM molecules were highly charged (e.g., at low $\mathrm{pH}$ ) and more counterions would be entrapped into their structure.

The orientation of the nanowires in the aggregates was also investigated near the IEPs by recording TEM images on samples prepared in the same way as for the mobility and scattering experiments (Figure 3). Accordingly, aggregating TiONW samples were imaged in the presence of G2 (Figure 3a), G6 (Figure 3b), and G10 (Figure 3c) dendrimers. It can be clearly seen that the nanowires prefer face-to-face orientation in the aggregates which leads to the formation of bundles or "spaghetti-like" structure regardless of the molecular weight and generation of the PAMAMs. The same orientation of the TiONWs was observed in AFM images during aggregation induced by inert salts or other type of polyelectrolytes. ${ }^{20}$ The dark spots on the TEM images may originate from the presence of anatase nanocrystals grown on the nanowires, but the resolution of our instrument did not allow us to confirm this unambiguously.

Effects of lonic Strength. Charging and aggregation were studied at different ionic strengths in the TiONW-G6 system first. Although the electrophoretic mobilities follow the general trend as described in the previous section, change in the ionic strength affects the shape of the curves significantly (Figure 4a). At low dendrimer dose, the mobility values are constant and refer to the electrophoretic mobility of the bare particles at the corresponding ionic strength (Figure 5a). Such values are very similar at lower salt levels $(1$ and $10 \mathrm{mM})$, while they decreased in magnitude at higher ionic strength $(100 \mathrm{mM})$ due to the screening effect of the counterions on the particle surface charge. A similar phenomenon was observed at high doses where the particles are positively charged due to the charge reversal process. Accordingly, the mobility values are smaller at the ASP at high ionic strength, but the change is less significant compared to the low dendrimer dose case. Furthermore, the PAMAM doses at the IEP shift toward higher concentrations, indicating a counterion condensation into the adsorbed dendrimer structure. The same shift was also experienced with other weakly charged polyelectrolytes during their adsorption of oppositely charged particles. ${ }^{59}$

The ionic strength had a huge influence on the aggregation of the TiONWs in the presence of the G6 dendrimers (Figure $4 \mathrm{~b})$. As discussed earlier, the stability ratios at $1 \mathrm{mM} \mathrm{KCl}$ concentration indicate stable dispersions at low and high PAMAM doses, while fast aggregation occurs in the intermediate regime near the IEP. The shape of the stability ratio versus dose curves changed dramatically at higher ionic strengths (10 and $100 \mathrm{mM})$. The TiONWs aggregate rapidly in the entire range at and below the IEP. This fact is not surprising since the critical coagulation concentration (CCC, which
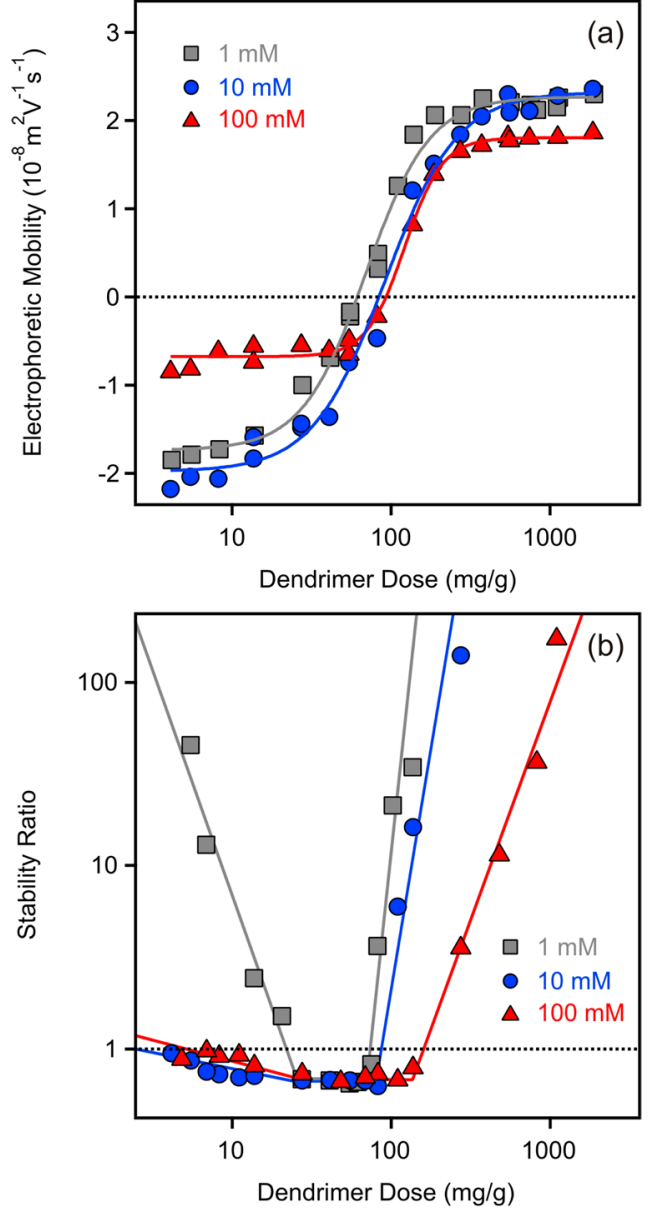

Figure 4. Electrophoretic mobility (a) and stability ratio (b) values as a function of the dendrimer dose measured in dispersions of $7 \mathrm{mg} / \mathrm{L}$ TiONW and G6 at different ionic strengths adjusted by $\mathrm{KCl}$. The lines are just to guide the eye.

separates fast and slow aggregation regimes) was determined as $8 \mathrm{mM}$ for the bare nanowires. ${ }^{22}$ Accordingly, only a small amount of PAMAM is adsorbed on the TiONWs at low doses and, hence, the aggregation rate corresponds to the value of the bare particles (Figure 5b). However, stable dispersions and slow aggregation were observed at high dendrimer concentration where the charge reversal resulted in nanowires of positive charge. The slopes at higher dose are also sensitive to the ionic strength due to the screening effect of the counterions on the surface. In these cases, the counterions are the negative chloride ions and such a screening effect was also discovered in the electrophoretic mobility measurements (Figure 4a) as discussed above. The stability ratios are below 1 at all ionic strengths around the IEP. The lack of the effect of salt level on the responsible patch-charge interaction indicates that the adsorbed amount and the structure of the dendrimer layers on the surface are not affected by the $\mathrm{KCl}$ concentration; therefore, the ionic strength dependence of the stability curves is only due to the electrostatic screening of the counterions on the particle surfaces especially at higher doses. This set of data shed light on the stabilization of the dispersions by PAMAM adsorption even at higher ionic strengths where the bare particles rapidly aggregate indicated by their low CCC values.

To further explore this issue, surface charges and aggregation were studied with nanowires coated with dendrimers of different generations. Accordingly, a PAMAM dose higher 

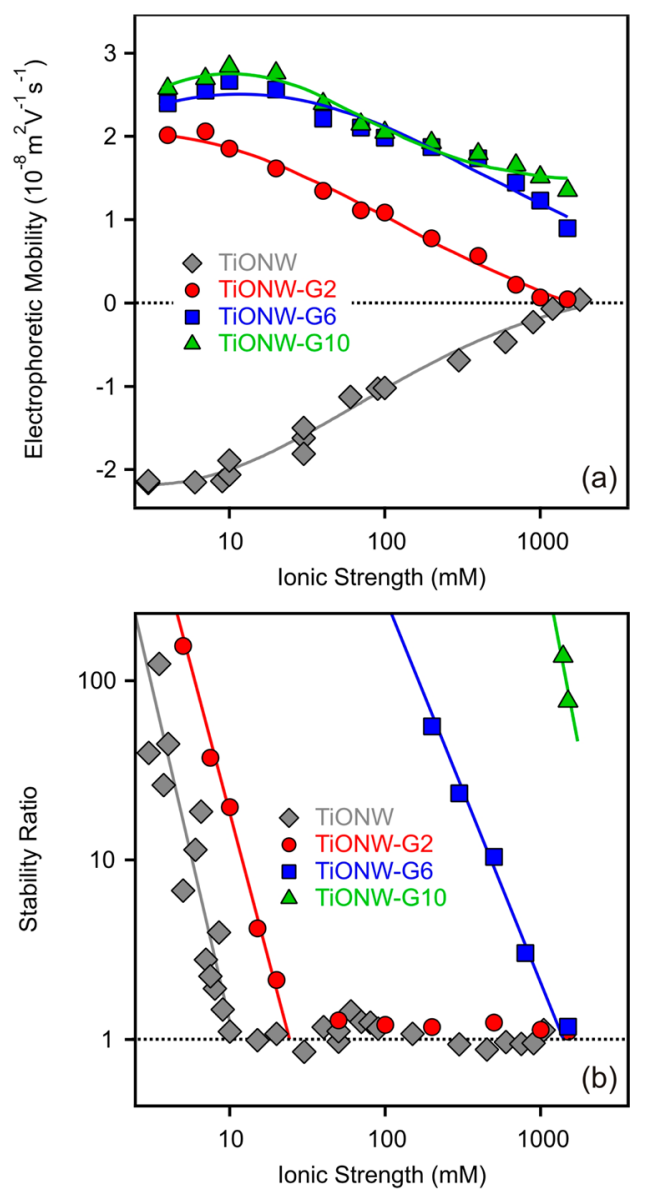

Figure 5. Electrophoretic mobility (a) and stability ratio (b) values of bare (TiONW) and PAMAM dendrimer-coated nanowires using different generations of PAMAM (TiONW-G2, TiONW-G6, and TiONW-G10) as a function of the ionic strength adjusted by $\mathrm{KCl}$ at $\mathrm{pH}$ 9. For the coated particles, about $1000 \mathrm{mg} / \mathrm{g}$ dendrimer doses were used to cover the TiONWs. The solid lines are to guide the eye.

than the onset of the ASP was applied in order to completely cover the TiONW surfaces and to obtain positively charged TiONW-G2, TiONW-G6, and TiONW-G10 particles. Figure 5a shows the electrophoretic mobilities of such nanowires together with the bare TiONW over a wide range of ionic strengths. The trends observed with the bare and TiONW-G2 particles are very similar, but of opposite sign of the charge due to the charge reversal process. The magnitude of the mobilities decreased with increasing the ionic strength and met around zero at high electrolyte concentration. This fact indicates that both bare and G2-coated nanowires has the same charge at an elevated $\mathrm{KCl}$ level, shedding light on the desorption of the G2 dendrimers from the particle surface. Such reversible adsorption has already been observed with low generation dendrimers ${ }^{38,42}$ and also with other multivalent ions. ${ }^{60}$ The electrophoretic mobilites of TiONW-G6 and TiONW-G10 nanowires were found to be very similar to slightly higher values in the latter case. Accordingly, the positive mobility values were recorded in the entire ionic strength range investigated and they decreased with increasing electrolyte concentration. The electrophoretic mobilities remained significantly positive even at high $\mathrm{KCl}$ concentrations where the mobility of the TiONW-G2 has already reached zero. The decrease of mobilities in the case of higher generation systems is most likely due to the screening effect of the chloride anions on the positive surface charge.

On the basis of these results, we have tested the aggregation processes (Figure $5 b$ ) under the same experimental conditions ( $\mathrm{pH}$, ionic strength range, TiONW concentration, and dendrimer dose). The TiONW and TiONW-G2 systems showed similar behavior with a small shift in the CCC. As one could predict applying the DLVO theory, the particles were stable at low ionic strength due to the repulsive forces originating from the overlap of the electric double layers. By increasing the salt level, the surface charges are screened and such double layer repulsion disappears leading to the predominance of the attractive van der Waals forces and to rapidly aggregating samples. The mobility results (Figure 5a) indicate that this screening process is accompanied by desorption of the G2 molecules. Since the CCCs are slightly different, desorption of the dendrimers is incomplete at the ionic strength corresponding to the range of the CCCs.

In addition, the stability curves of TiONW-G6 and TiONW-G10 nanowires were also sensitive to the $\mathrm{KCl}$ concentration: stability ratios decreased with increasing salt level (Figure 5b). For the first system, a CCC of about 1500 $\mathrm{mM}$ was found, indicating an enormous stabilizing effect of the dendrimer coating compared to the $8 \mathrm{mM}$ value determined for the bare particles. Moreover, the stabilization effect was even higher in the case of TiONW-G10, where only slow aggregation was observed at high salt levels and we were not able to reach the CCC due to the solubility limit of $\mathrm{KCl}$. If one compares these data to the lower generation TiONW-G2 system, the following conclusions can be made. First, all coated systems are stable at very low ionic strengths regardless of the generation of the PAMAMs. Second, increasing the ionic strength led to desorption of G2 which is most likely not favorable for $\mathrm{G} 6$ and $\mathrm{G} 10$ even at high $\mathrm{KCl}$ concentrations. These facts clearly indicate that higher generation dendrimers can be used as dispersing agents even at high ionic strength; however, G2 most likely desorbs under these experimental conditions. For instance, in drug or gene delivery systems where the ionic strength is over $100 \mathrm{mM}$ and hence aggregation of the bare nanowires would certainly occur, PAMAM dendrimer coating prevents such aggregation and provides highly stable dispersions suitable for the delivery and release processes.

\section{CONCLUSIONS}

Electrophoretic, time-resolved DLS, and TEM experiments were carried out to clarify the effect of PAMAM dendrimers of different generations on the colloidal stability of TiONW dispersions. Our study on charging revealed that PAMAMs strongly adsorbed on the oppositely charged particles and charge neutralization as well as reversal occurred. These phenomena were independent of the molecular weight of the macromolecules. The dispersions were stable at low and high dendrimer doses at low ionic strength where the particles possessed sufficiently high negative or positive charge, respectively. Rapid aggregation and unstable systems were obtained near the dose of the charge reversal point. The predominating interparticle forces were mainly of DLVO origin; nevertheless, additional attraction due to patch-charge interactions was also discovered. The orientation of the nanowires in the aggregates resulted in the formation of bundles or "spaghetti-like" structures regardless of the generation of the dendrimers. The ionic strength dependence 
of the aggregation rates pointed out an enormous stabilization effect of the PAMAM macromolecules of higher generations since the coated TiONW dispersions were stable even at salt levels where the bare particles undergo rapid aggregation due to their low CCC value. In conclusion, a potential nanocarrier (TiONW) to be used in biomedical applications can be stabilized by PAMAM macromolecules of higher generations and, on the basis of our results, appropriate doses can be calculated to obtain highly stable dispersions to be further investigated as biocompatible delivery systems. Accordingly, TiONWs coated with higher generation dendrimers are potential candidates in delivery processes as carriers where unwanted aggregation is prevented by the PAMAM layer even under extremely high ionic strengths.

\section{AUTHOR INFORMATION}

\section{Corresponding Author}

*E-mail: istvan.szilagyi@unige.ch.

\section{Author Contributions}

The manuscript was written through contributions of all authors. All authors have given approval to the final version of the manuscript.

\section{Notes}

The authors declare no competing financial interest.

\section{ACKNOWLEDGMENTS}

Financial support from the Swiss National Science Foundation (150162), Swiss Scientific Exchange Program (14033), University of Geneva, Swiss Contribution ( $\mathrm{SH} / 7 / 2 / 20$ ), Swiss Secretariat for Education, Research and Innovation (C15.0024), and COST Actions MP1106 and CM1303 is gratefully acknowledged. M.P. and I.S. are grateful to Prof. Michal Borkovec for providing access to the light scattering instruments in his laboratory. The authors also thank Mr. Olivier Vassalli for the technical support during the DLS experiments.

\section{REFERENCES}

(1) Nicolosi, V.; Chhowalla, M.; Kanatzidis, M. G.; Strano, M. S.; Coleman, J. N. Liquid Exfoliation of Layered Materials. Science 2013, 340, 1226419.

(2) Bai, Y.; Xing, Z.; Yu, H.; Li, Z.; Amal, R.; Wang, L. Z. Porous Titania Nanosheet/Nanoparticle Hybrids as Photoanodes for DyeSensitized Solar Cells. ACS Appl. Mater. Interfaces 2013, 5, 1205812065.

(3) Pusztai, P.; Puskas, R.; Varga, E.; Erdohelyi, A.; Kukovecz, A.; Konya, Z.; Kiss, J. Influence of Gold Additives on the Stability and Phase Transformation of Titanate Nanostructures. Phys. Chem. Chem. Phys. 2014, 16, 26786-26797.

(4) Potari, G.; Madarasz, D.; Nagy, L.; Laszlo, B.; Sapi, A.; Oszko, A.; Kukovecz, A.; Erdohelyi, A.; Konya, Z.; Kiss, J. Rh-Induced Support Transformation Phenomena in Titanate Nanowire and Nanotube Catalysts. Langmuir 2013, 29, 3061-3072.

(5) Madarasz, D.; Potari, G.; Sapi, A.; Laszlo, B.; Csudai, C.; Oszko, A.; Kukovecz, A.; Erdohelyi, A.; Konya, Z.; Kiss, J. Metal Loading Determines the Stabilization Pathway for $\mathrm{Co}^{2+}$ in Titanate Nanowires: Ion Exchange vs. Cluster Formation. Phys. Chem. Chem. Phys. 2013, 15, 15917-15925.

(6) Osada, M.; Sasaki, T. Two-Dimensional Dielectric Nanosheets: Novel Nanoelectronics From Nanocrystal Building Blocks. Adv. Mater. 2012, 24, 210-228.

(7) Koka, A.; Sodano, H. A. High-sensitivity Accelerometer Composed of Ultra-long Vertically Aligned Barium Titanate Nanowire Arrays. Nat. Commun. 2013, 4, 2682.
(8) Bavykin, D. V.; Walsh, F. C. Elongated Titanate Nanostructures and Their Applications. Eur. J. Inorg. Chem. 2009, 2009, 977-997.

(9) Zhou, W. J.; Liu, X. J.; Sang, Y. H.; Zhao, Z. H.; Zhou, K.; Liu, H.; Chen, S. W. Enhanced Performance of Layered Titanate NanowireBased Supercapacitor Electrodes by Nickel Ion Exchange. ACS Appl. Mater. Interfaces 2014, 6, 4578-4586.

(10) Wang, Q. G.; Gao, Q. M.; Shi, J. L. Enhanced Catalytic Activity of Hemoglobin in Organic Solvents by Layered Titanate Immobilization. J. Am. Chem. Soc. 2004, 126, 14346-14347.

(11) Liu, H.; Duan, C. Y.; Su, X.; Dong, X. N.; Huang, Z.; Shen, W. Q.; Zhu, Z. F. A Hemoglobin Encapsulated Titania Nanosheet Modified Reduced Graphene Oxide Nanocomposite as a Mediatorfree Biosensor. Sens. Actuators, B 2014, 203, 303-310.

(12) Soh, N.; Kaneko, S.; Uozumi, K.; Ueda, T.; Kamada, K. Preparation of an Enzyme/inorganic Nanosheet/magnetic Bead Complex and its Enzymatic Activity. J. Mater. Sci. 2014, 49, 80108015.

(13) Ding, X. L.; Yang, X. Q.; Zhou, L.; Lu, H. B.; Li, S. B.; Gao, Y.; Lai, C. H.; Jiang, Y. Titanate Nanowire Scaffolds Decorated with Anatase Nanocrystals Show Good Protein Adsorption and Low Cell Adhesion Capacity. Int. J. Nanomed. 2013, 8, 569-579.

(14) Papa, A. L.; Dumont, L.; Vandroux, D.; Millot, N. Titanate Nanotubes: Towards a Novel and Safer Nanovector for Cardiomyocytes. Nanotoxicology 2013, 7, 1131-1142.

(15) Zheng, X. T.; He, H. L.; Li, C. M. Multifunctional Graphene Quantum Dots-conjugated Titanate Nanoflowers for Fluorescencetrackable Targeted Drug Delivery. RSC Adv. 2013, 3, 24853-24857.

(16) Magrez, A.; Horvath, L.; Smajda, R.; Salicio, V.; Pasquier, N.; Forro, L.; Schwaller, B. Cellular Toxicity of $\mathrm{TiO}_{2}$-Based Nanofilaments. ACS Nano 2009, 3, 2274-2280.

(17) Papa, A. L.; Boudon, J.; Bellat, V.; Loiseau, A.; Bisht, H.; Sallem, F.; Chassagnon, R.; Berard, V.; Millot, N. Dispersion of Titanate Nanotubes for Nanomedicine: Comparison of PEI and PEG Nanohybrids. Dalton Trans. 2015, 44, 739-746.

(18) Beke, S.; Korosi, L.; Scarpellini, A.; Anjum, F.; Brandi, F. Titanate Nanotube Coatings on Biodegradable Photopolymer Scaffolds. Mater. Sci. Eng., C 2013, 33, 2460-2463.

(19) Papa, A. L.; Maurizi, L.; Vandroux, D.; Walker, P.; Millot, N. Synthesis of Titanate Nanotubes Directly Coated with USPIO in Hydrothermal Conditions: A New Detectable Nanocarrier. J. Phys. Chem. C 2011, 115, 19012-19017.

(20) Horváth, E.; Grebikova, L.; Maroni, P.; Szabó, T.; Magrez, A.; Forró, L.; Szilagyi, I. Dispersion Characteristics and Aggregation in Titanate Nanowire Colloids. ChemPlusChem 2014, 79, 592-600.

(21) Szabo, T.; Toth, V.; Horvath, E.; Szilagyi, I. Formulation of Multifunctional Material Dispersions. Chimia 2014, 68, 454-454.

(22) Szabo, T.; Toth, V.; Horvath, E.; Forro, L.; Szilagyi, I. Tuning the Aggregation of Titanate Nanowires in Aqueous Dispersions. Langmuir 2015, 31, 42-49.

(23) Parimi, S.; Barnes, T. J.; Callen, D. F.; Prestidge, C. A. Mechanistic Insight into Cell Growth, Internalization, and Cytotoxicity of PAMAM Dendrimers. Biomacromolecules 2010, 11, 382-389.

(24) Astruc, D.; Boisselier, E.; Ornelas, C. Dendrimers Designed for Functions: From Physical, Photophysical and Supramolecular Properties to Applications in Sensing, Catalysis, Molecular Electronics, Photonics, and Nanomedicine. Chem. Rev. 2010, 110, 1857-1959.

(25) Liu, X. H.; Ma, D. M.; Tang, H.; Tan, L.; Xie, Q. J.; Zhang, Y. Y.; Ma, M.; Yao, S. Z. Polyamidoamine Dendrimer and Oleic AcidFunctionalized Graphene as Biocompatible and Efficient Gene Delivery Vectors. ACS Appl. Mater. Interfaces 2014, 6, 8173-8183.

(26) Frechet, J. M. J.; Tomalia, D. A. Dendrimers and Other Dendritic Polymers; John Wiley: New York, 2001.

(27) Kirkorian, K.; Ellis, A.; Twyman, L. J. Catalytic Hyperbranched Polymers as Enzyme Mimics; Exploiting the Principles of Encapsulation and Supramolecular Chemistry. Chem. Soc. Rev. 2012, 41, 6138-6159.

(28) Cakara, D.; Kleimann, J.; Borkovec, M. Microscopic Protonation Equilibria of Poly(amidoamine) Dendrimers from Macroscopic Titrations. Macromolecules 2003, 36, 4201-4207. 
(29) Banyai, I.; Keri, M.; Nagy, Z.; Berka, M.; Balogh, L. P. Selfdiffusion of Water and Poly(amidoamine) Dendrimers in Dilute Aqueous Solutions. Soft Matter 2013, 9, 1645-1655.

(30) Jachimska, B.; Lapczynska, M.; Zapotoczny, S. Reversible Swelling Process of Sixth-generation Poly(amido amine) Dendrimers Molecule as Determined by Quartz Crystal Microbalance Technique. J. Phys. Chem. C 2013, 117, 1136-1145.

(31) Cheng, Y. Y.; Qu, H.; Ma, M. L.; Xu, Z. H.; Xu, P.; Fang, Y. J.; $\mathrm{Xu}, \mathrm{T}$. W. Polyamidoamine (PAMAM) Dendrimers as Biocompatible Carriers of Quinolone Antimicrobials: An in vitro study. Eur. J. Med. Chem. 2007, 42, 1032-1038.

(32) Luo, D.; Haverstick, K.; Belcheva, N.; Han, E.; Saltzman, W. M. Poly(ethylene glycol)-Conjugated PAMAM Dendrimer for Biocompatible, High-efficiency DNA Delivery. Macromolecules 2002, 35, $3456-3462$

(33) Tully, D. C.; Frechet, J. M. J. Dendrimers at Surfaces and Interfaces: Chemistry and Applications. Chem. Commun. 2001, 12291239.

(34) dos Reis, M. J.; Prevot, V.; Leroux, F.; Silverio, F.; Valim, J. B. Dendrimer Intercalation in Layered Double Hydroxides. J. Porous Mater. 2010, 17, 443-451.

(35) Leisner, D.; Imae, T. Structural Evolution of an Interpolyelectrolyte Complex of Charged Dendrimers Interacting with Poly(Lglutamate). J. Phys. Chem. B 2004, 108, 1798-1804.

(36) Ott, L. S.; Finke, R. G. Transition-metal Nanocluster Stabilization for Catalysis: A Critical Review of Ranking Methods and Putative Stabilizers. Coord. Chem. Rev. 2007, 251, 1075-1100.

(37) Muresan, L.; Maroni, P.; Popa, I.; Porus, M.; Longtin, R.; Papastavrou, G.; Borkovec, M. Probing Conformational Changes of Polyamidoamine (PAMAM) Dendrimers Adsorbed on Silica Substrates. Macromolecules 2011, 44, 5069-5071.

(38) Longtin, R.; Maroni, P.; Borkovec, M. Transition from Completely Reversible to Irreversible Adsorption of Poly(amido amine) Dendrimers on Silica. Langmuir 2009, 25, 2928-2934.

(39) Muller, T.; Yablon, D. G.; Karchner, R.; Knapp, D.; Kleinman, M. H.; Fang, H.; Durning, C. J.; Tomalia, D. A.; Turro, N. J.; Flynn, G. W. AFM Studies of High-generation PAMAM Dendrimers at the Liquid/solid Interface. Langmuir 2002, 18, 7452-7455.

(40) Rahman, K. M. A.; Durning, C. J.; Turro, N. J.; Tomalia, D. A. Adsorption of Poly(amidoamine) Dendrimers on Gold. Langmuir 2000, 16, 10154-10160.

(41) Zheng, J.; Stevenson, M. S.; Hikida, R. S.; Van Patten, P. G. Influence of $\mathrm{pH}$ on Dendrimer-protected Nanoparticles. J. Phys. Chem. B 2002, 106, 1252-1255.

(42) Lin, W.; Galletto, P.; Borkovec, M. Charging and Aggregation of Latex Particles by Oppositely Charged Dendrimers. Langmuir 2004, 20, 7465-7473.

(43) Popa, I.; Papastavrou, G.; Borkovec, M. Charge Regulation Effects on Electrostatic Patch-charge Attraction Induced by Adsorbed Dendrimers. Phys. Chem. Chem. Phys. 2010, 12, 4863-4871.

(44) Popa, I.; Papastavrou, G.; Borkovec, M.; Trulsson, M.; Jonsson, B. Long-ranged Attractive Forces Induced by Adsorbed Dendrimers: Direct Force Measurements and Computer Simulations. Langmuir 2009, 25, 12435-12438.

(45) Finessi, M.; Szilagyi, I.; Maroni, P. Dendrimer Induced Interaction Forces Between Colloidal Particles Revealed by Direct Force and Aggregation Measurements. J. Colloid Interface Sci. 2014, 417, 346-355.

(46) Niecikowska, A.; Krasowska, M.; Ralston, J.; Malysa, K. Role of Surface Charge and Hydrophobicity in the Three-phase Contact Formation and Wetting Film Stability under Dynamic Conditions. J. Phys. Chem. C 2012, 116, 3071-3078.

(47) Kuscer, D.; Stavber, G.; Trefalt, G.; Kosec, M. Formulation of an Aqueous Titania Suspension and its Patterning with Ink-Jet Printing Technology. J. Am. Ceram. Soc. 2012, 95, 487-493.

(48) Hassan, P. A.; Rana, S.; Verma, G. Making Sense of Brownian Motion: Colloid Characterization by Dynamic Light Scattering. Langmuir 2015, 31, 3-12.
(49) Quesada-Perez, M.; Gonzalez-Tovar, E.; Martin-Molina, A.; Lozada-Cassou, M.; Hidalgo-Alvarez, R. Overcharging in Colloids: Beyond the Poisson-Boltzmann Approach. ChemPhysChem 2003, 4 234-248.

(50) Lyklema, J. Overcharging, Charge Reversal: Chemistry or Physics? Colloids Surf., A 2006, 291, 3-12.

(51) Faraudo, J.; Martin-Molina, A. Competing Forces in the Interaction of Polyelectrolytes with Charged Interfaces. Curr. Opin. Colloid Interface Sci. 2013, 18, 517-523.

(52) Schneider, C.; Hanisch, M.; Wedel, B.; Jusufi, A.; Ballauff, M. Experimental Study of Electrostatically Stabilized Colloidal Particles: Colloidal Stability and Charge Reversal. J. Colloid Interface Sci. 2011, $358,62-67$.

(53) Zaccone, A.; Wu, H.; Lattuada, M.; Morbidelli, M. Correlation Between Colloidal Stability and Surfactant Adsorption/association Phenomena Studied by Light Scattering. J. Phys. Chem. B 2008, 112, 1976-1986.

(54) Chen, K. L.; Mylon, S. E.; Elimelech, M. Enhanced Aggregation of Alginate-coated Iron Oxide (Hematite) Nanoparticles in the Presence of, Calcium, Strontium and Barium Cations. Langmuir 2007, 23, 5920-5928.

(55) Elimelech, M.; Gregory, J.; Jia, X.; Williams, R. A. Particle Deposition and Aggregation: Measurement, Modeling, and Simulation; Butterworth-Heinemann Ltd.: Oxford, U.K., 1995.

(56) Evans, D. F.; Wennerstrom, H. The Colloidal Domain; John Wiley: New York, 1999.

(57) Szilagyi, I.; Trefalt, G.; Tiraferri, A.; Maroni, P.; Borkovec, M. Polyelectrolyte Adsorption, Interparticle Forces, and Colloidal Aggregation. Soft Matter 2014, 10, 2479-2502.

(58) Leong, Y. K. Interparticle Forces Arising from an Adsorbed Strong Polyelectrolyte in Colloidal Dispersions: Charged Patch Attraction. Colloid Polym. Sci. 1999, 277, 299-305.

(59) Szilagyi, I.; Rosicka, D.; Hierrezuelo, J.; Borkovec, M. Charging and Stability of Anionic Latex Particles in the Presence of Linear Poly(ethylene imine). J. Colloid Interface Sci. 2011, 360, 580-585.

(60) Szilagyi, I.; Polomska, A.; Citherlet, D.; Sadeghpour, A.; Borkovec, M. Charging and Aggregation of Negatively Charged Colloidal Latex Particles in the Presence of Multivalent Oligoamine Cations. J. Colloid Interface Sci. 2013, 392, 34-41. 